\title{
ЭКОНОМИКО-МАТЕМАТИЧЕСКАЯ МОДЕЛЬ РАСПРЕДЕЛЕНИЯ РАБОТ В ВИРТУАЛЬНОЙ ОРГАНИЗАЦИИ
}

\author{
(c) 2018 Хромов Иван Евгеньевич \\ кандидат экономических наук, старший научный сотрудник \\ Центральный экономико-математический институт РАН \\ 117418, г. Москва, Нахимовский проспект, 47 \\ E-mail:khromov_gaugn@mail.ru
}

В настоящее время развивается концепция виртуальной организации как новой организационной формы управления предприятиями. Для создания виртуальной организации необходим правильный выбор партнёров, а для эффективного управления созданной организацией необходимо грамотно распределить работы между отобранными партнёрами. В данной работе описана модель, позволяющая решить данные задачи при помощи экономико-математических методов.

Ключевые слова: виртуальная организация, организационная структура, партнёрство, сокращение издержек, управление предприятием, проекты, финансовое планирование, закупки, экономико-математическое моделирование, задача распределения, критерии, ограничения, задача о назначениях, матрица назначений, сетевой график.

Для создания виртуальной организации необходим правильный выбор партнеров, а для эффективного управления созданной организацией необходимо распределить работы между отобранными партерами оптимальным образом.

Целью заказчика, который является центром организации, является размещение контракта на работу с минимальными затратами $\sum_{j=1}^{m} \lambda_{j} x_{j}$, а целью любого из подрядчиков - максимизация полученной ими прибыли. Прибыль партера определяется как разность между полученным от заказчика вознаграждением и собственными затратами.

Таким образом, в случае виртуальной организации, целью оптимизации будет выступать не степень удовлетворения потребностей конечного потребителя, а минимизация затрат или максимизация прибыли центра или партнеров.

Кроме того, при создании виртуальной организации необходимо также учитывать следующие условия:

1. Условия и критерии отбора исполнителей работ должны быть четко установлены, известны и понятны всем возможным подрядчикам.

2. В процессе распределения работ наиболее эффективным является минимально участие и влияния центра или заказчиков. Это необходимо для устранения субъективных предпочтений заказчика.

Можно выделить три вида задач распределе- ния работ в рамках проекта в виртуальной организации. Общим для этих задач является выбор и оценка наиболее подходящего исполнителя для каждой работы.

1. Задача выбора подрядчика, который может наилучшим образом выполнить поступивший заказ.

2. Задача оптимального распределения совокупности партнеров для выполнения ряда работ, при условии, что одна работа полностью выполняется только одним агентом.

3. Задача оптимального распределения работ по подрядчикам, если одна работа требует для выполнения участия сразу нескольких подрядчиков.

Также можно выделить группы характеристик, которые могут выступать и как ограничения и как критерии:

1. Временные характеристики работы

2. Стоимостные характеристики работы

3. Качество выполнения работы

По этим критериям заказчик может вводить ограничения при первоначальном отборе потенциальных исполнителей работ, также эти же критерии могут использоваться при оптимизации распределения работ между выбранной совокупностью партнеров. Впрочем, в ситуации, когда у нескольких возможных партнеров параметры выполнения, возможно использование других параметров. Например, это может быть уровень компетенции подрядчика, поскольку он 
может выступать как критерии качества выполнения работы.

Таким образом, при оптимизации проекта, то есть при выборе подрядчика необходимо как минимизировать время и стоимость выполнения работы, так и максимизировать качество выполнения работы.

Задача распределения совокупности работ по совокупности партнеров может быть представлена как задача о назначениях. Однако, в рамках задачи о назначениях можно говорить только при условии заранее отобранного пула подрядчиков - например в рамках закрытого тендера. Задача о назначениях является частным случаем транспортной задачи. Так как постановка классической задачи состоит в нахождении пар «Работа - Исполнителей», которые обеспечивают минимум суммарных затрат на выполнение всего проекта, и каждый исполнитель может исполнять только одну работу, равно как для выполнения одной работы требуется только один исполнитель, то это также накладывает ограничения на задачу. Это значит, что в проекте заранее должно быть оговорено данное условие - исполнитель не может выполнять более одной работы.

Общий вид модели задачи о назначениях

$$
\begin{gathered}
\mathrm{L}(\mathrm{X})=\sum_{i=1}^{n} \sum_{j=1}^{m} c_{i j} x_{i j} \rightarrow \min \\
\left\{\begin{array}{c}
\sum_{j=1}^{m} x_{i j}=1(i=\overline{1, n}) \\
\sum_{i=1}^{n} x_{i j}=1(j=\overline{1, m}) \\
x_{i j}=\left\{\begin{array}{l}
0, \\
1,
\end{array}(i=\overline{1, n} ; j=\overline{1, m})\right.
\end{array}\right.
\end{gathered}
$$

Исходные параметры модели задачи о назначениях:

1. $\mathrm{n}$ - количество исполнителей, $\mathrm{m}-$ количество работ;

2. $\mathrm{a}_{\mathrm{i}}=1$ - единичное количество ресурса $\mathrm{A}_{\mathrm{i}}$ (i = 1, n). Например, один исполнитель

3. $\mathrm{b}_{\mathrm{j}}=1$ - единичное количество работы $\mathrm{B}_{\mathrm{j}}$ $(\mathrm{j}=1, \mathrm{~m})$. Например, одна работа.

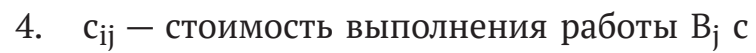
помощью ресурса $\mathrm{A}_{\mathrm{i}}$. Например, $\mathrm{c}_{\mathrm{ij}}$ может быть компетентностью i-го исполнителя при выполнении ј-й работы, или стоимость выполнения работы, или время выполнения работы.

\section{Искомые параметры}

1. $x_{i j}$ - факт назначения или «не назначения» исполнителя $A_{i}$ на работу $B_{j}$ :

$x_{i j}=\left\{\begin{array}{c}0, \text { если } i-\text { й ресурс не назначен на } j-\text { ю работу } \\ 1, \text { если } i-\text { й ресурс назначен на } j-\text { ю работу }\end{array}\right.$

2. $\mathrm{L}(\mathrm{X})$ - общая (суммарная) характеристика качества распределения исполнителей по работам.

Более важным для создания и функционирования виртуальной организации является оптимальный отбор партнеров. Отбор партнеров может проводиться в рамках открытого тендера, в котором могут принимать участие любые поставщики товаров или услуг.

В документации открытого тендера заранее определяются сроки выполнения работ, качество выполнения работы, максимальные стоимостные характеристики работ и прочие условия.

Так как каждый из потенциальных исполнителей предоставляет заказчику заявку с указанием стоимости и времени выполнения ими работ, то для определения совокупности подрядчиков, подходящих для выполнения проекта можно воспользоваться методом сжатия. Сжа-

\begin{tabular}{|c|c|c|c|c|c|}
\hline \multirow{2}{*}{$\begin{array}{c}\text { Исполнители, } \\
\mathrm{A}_{\mathrm{i}} \\
\end{array}$} & \multicolumn{4}{|c|}{ Работы, Bi } & \multirow{2}{*}{$\begin{array}{c}\text { Количество } \\
\text { исполнителей }\end{array}$} \\
\hline & $\mathrm{B}_{1}$ & $\mathrm{~B}_{2}$ & $\ldots$ & $\mathrm{B}_{\mathrm{m}}$ & \\
\hline $\mathrm{A}_{1}$ & $\mathrm{c}_{11}$ & $\mathrm{c}_{12}$ & $\ldots$ & $\mathrm{c}_{1 \mathrm{~m}}$ & 1 \\
\hline \multirow[t]{2}{*}{$\mathrm{A}_{2}$} & $\mathrm{c}_{21}$ & $\mathrm{c}_{22}$ & $\ldots$ & $\mathrm{c}_{2} \mathrm{~m}$ & 1 \\
\hline & $\ldots$ & $\ldots$ & $\ldots$ & $\ldots$ & $\ldots$ \\
\hline$A_{n}$ & $\mathrm{c}_{\mathrm{n} 1}$ & $c_{n 2}$ & $\ldots$ & $\mathrm{c}_{\mathrm{nm}}$ & 1 \\
\hline $\begin{array}{c}\text { Количество } \\
\text { работ }\end{array}$ & 1 & 1 & $\ldots$ & 1 & $\sum_{i=1}^{n} a_{i}=\sum_{j=1}^{m} b_{j}$ \\
\hline
\end{tabular}

Общий вид матрицы задачи о назначениях 
тие (Crashing) представляет собой метод сокращения продолжительности проекта за счет сокращения одной (или более) работ, находящихся на критическом пути проекта. Подобное сжатие достигается, как правило, при помощи использования дополнительных ресурсов, например, увеличения количества рабочих.

На рисунке 1 изображена графическая зависимость между стоимостью и продолжительностью работ. Точка В соответствует нормальной продолжительности работы, точка А - наиболее сокращённой продолжительности работы. При этом затраты в точке А считаются предельными и их дальнейшее увеличение практически не влияет на сокращение времени работы, а затраты в точке В являются нормальными. Линия, соединяющая точки А и В, показывает изменение затрат при сокращении времени работы на одну единицу.

Однако, при использовании концепции виртуальной организации для выполнения работы можно выбрать одного исполнителя из совокупности. На рисунке 2 так же показана зави- симость между стоимостью и продолжительностью работы. Однако, заказчик может выбрать между двумя исполнителями, для каждого из которых существуют параметры предельных затрат и максимального сокращения времени, и нормальных затрат и времени.

В таком случае, при сокращении продолжительности при помощи крэшинга, в начале необходимо выбрать линию, крайняя правая точка на которой соответствует минимальным затратам при максимальном времени. На рисунке 2 это точка С. Однако, если при использовании метода, время сокращается на величину большую, чем соответствующая точке Е, то необходимо перейти к линии изменения затрат второго исполнителя.

На рисунке 3 показано изменение продолжительности проекта в зависимости от затрат. При этом каждая точка графика соответствует определенному пулу исполнителей. Таким образом, заказчик, определяя или время, или затраты проекта, автоматически определяет совокупность исполнителей и их распределение по

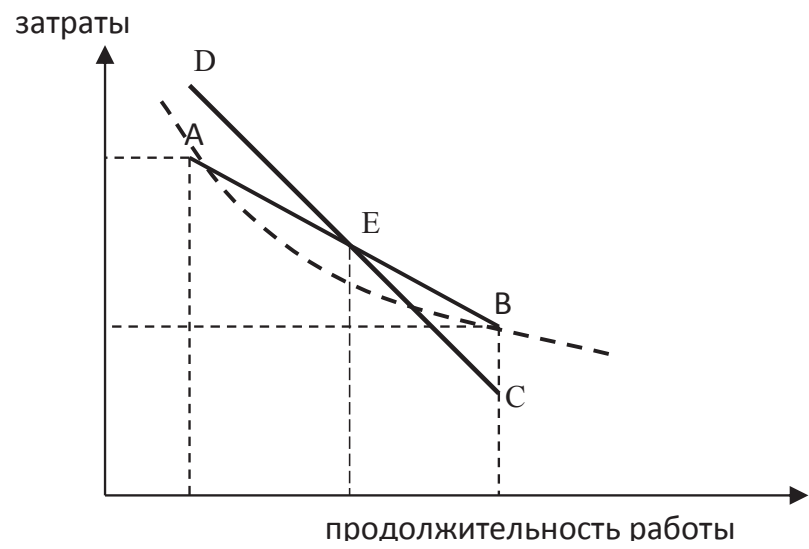

Puc. 2. Зависимость между продолжительностью работ и их стоимостью при наличии выбора исполнителя
Рис.1. Зависимость между продолжительностью работ и их стоимостью при отсутствии выбора исполнителя

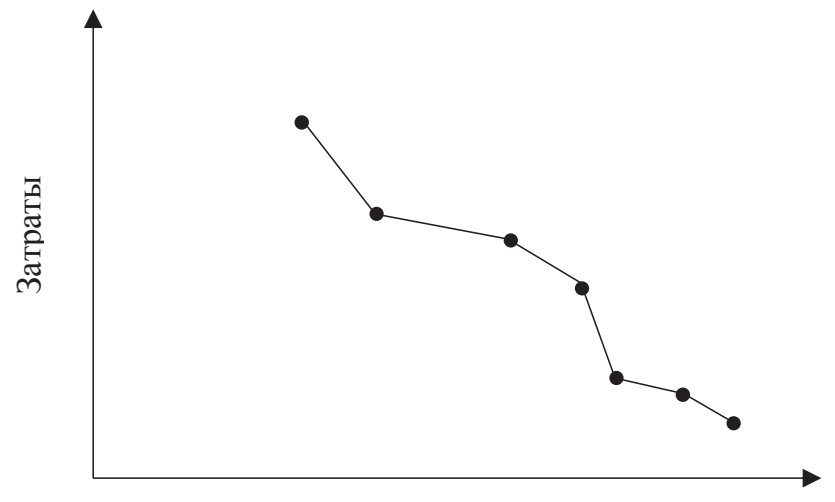

Продолжительность проекта

Puc. 3. Зависимость между продолжительностью работ и их стоимостью при выборе различных исполнителей для каждого этапа работ 
отдельным работам проекта.

Рассмотрим применение модели распределения работ по исполнителям на примере строительного подряда ОАО «РЖД» - «Строительства ливневой канализации с очистными сооружениями станции Ярославль-Главный» Северной железной дороги.

В процессе проведения тендера, компаниязаказчик ДКСС ОАО «РЖД» осуществляет выбор генерального субподрядчика так, чтобы её минимальные издержки были минимальными. То есть, в первую очередь учитываются стоимостные характеристики заказа, но не временные или качественные характеристики выполнения заказа.

Однако, если бы компания-заказчик использовала концепцию виртуальной организации, которая позволяет нескольким независимым компаниям временно объединяться в сеть партнеров, то, возможно, она могла бы сократить свои издержки.

Таблица 1 показывает исходные стоимостные характеристики каждого из возможных исполнителей. При решении задачи о назначении, каждому из исполнителей ставится в соответствие одна из работ так, чтобы минимизировать затраты на выполнение проекта.

При проведении тендеров, компания заказчик - ОАО «РЖД» выбирает единственного генерального подрядчика, который выполняет весь объем работ, минимизирую суммарные затраты на проект. Исходя из данных в таблице, это подрядчик № 1, сумма затрат, предложенных которым равна - 130548076 руб.

При использовании концепции виртуальной организации, в этом проекте, затраты будут меньше - 130546345 руб., так что данный проект возможно использовать данную концепцию. Однако, следует заметить, что сокращение издержек при применении модели распределения работ по исполнителям не обязательно и зависит от параметров стоимости исполнения работы отдельных исполнителей.

Однако, при проведении открытого тендера, необходимо в начале осуществить отбор партнеров.

Сначала построим сетевой график проекта (рис. 4), который отображает последовательность выполнения комплекса работ, с помощью которого можно отследить критический путь проекта.

Для использования метода сжатия (crashing), необходимы данные о предельных затрата и максимальном сокращении времени, и нормальных затратах и времени для каждого из потенциальных исполнителей работ. Кроме того, для каждой работы необходимо построить графики, отображающие взаимосвязь между стоимостью и продолжительностью работы.

Всего в проекте есть 8 работ: технологические решения (А), силовое электрооборудование (B), подготовка территории строительства (C), конструктивные решения и объемно-планировочные решения (D), наружные сети канализации (E), конструкции железобетонные (F), электроснабжение $(\mathrm{G})$, благоустройство $(\mathrm{H})$.

Кроме того, для каждого графика необходимо построить огибающую функцию. Огибающая функция является линейно-кусочной и состоит из минимумов каждого графика взаимосвязей. При сокращении времени работы, движение будет осуществляться по линии этой функции.

Таблица 1. Затраты на строительство ливневой канализации с очистными сооружениями станции Ярославль-Главный Северной железной дороги

\begin{tabular}{|c|c|c|c|c|c|c|c|c|c|}
\hline & 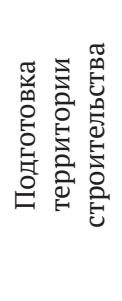 & 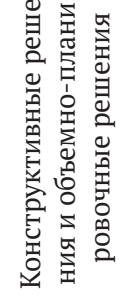 & 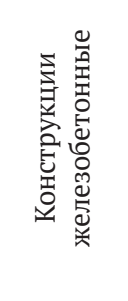 & 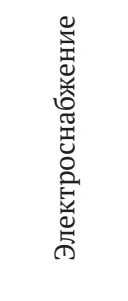 & 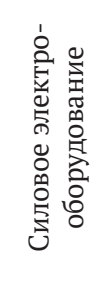 & 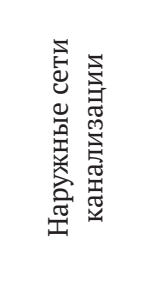 & 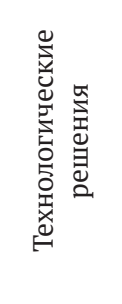 & 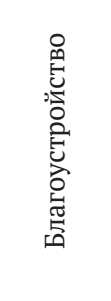 & 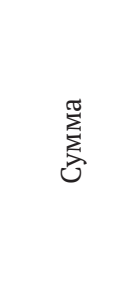 \\
\hline Подрядчик 1 & 5332 & 3249352 & 3817022 & 1248198 & 529632 & 117159470 & 4392473 & 146597 & 130548076 \\
\hline Подрядчик 2 & 5694 & 3250095 & 3817134 & 1248157 & 529429 & 117159628 & 4392548 & 146973 & 130549658 \\
\hline Подрядчик 3 & 5497 & 3248194 & 3817292 & 1248911 & 529893 & 117160872 & 4392542 & 147156 & 130550357 \\
\hline Подрядчик 4 & 5352 & 3250223 & 3817848 & 1248224 & 529049 & 117159629 & 4392557 & 146427 & 130549309 \\
\hline Подрядчик 5 & 5643 & 3249062 & 3817328 & 1248524 & 529310 & 117160408 & 4392524 & 146810 & 130549609 \\
\hline Подрядчик 6 & 5566 & 3249049 & 3817250 & 1248222 & 529552 & 117159582 & 4392755 & 147034 & 130549010 \\
\hline Подрядчик 7 & 5237 & 3249116 & 3817378 & 1248305 & 529911 & 117160376 & 4392632 & 146615 & 130549570 \\
\hline Подрядчик 8 & 5377 & 3250297 & 3817230 & 1248839 & 529676 & 117159388 & 4392466 & 146635 & 130549908 \\
\hline
\end{tabular}




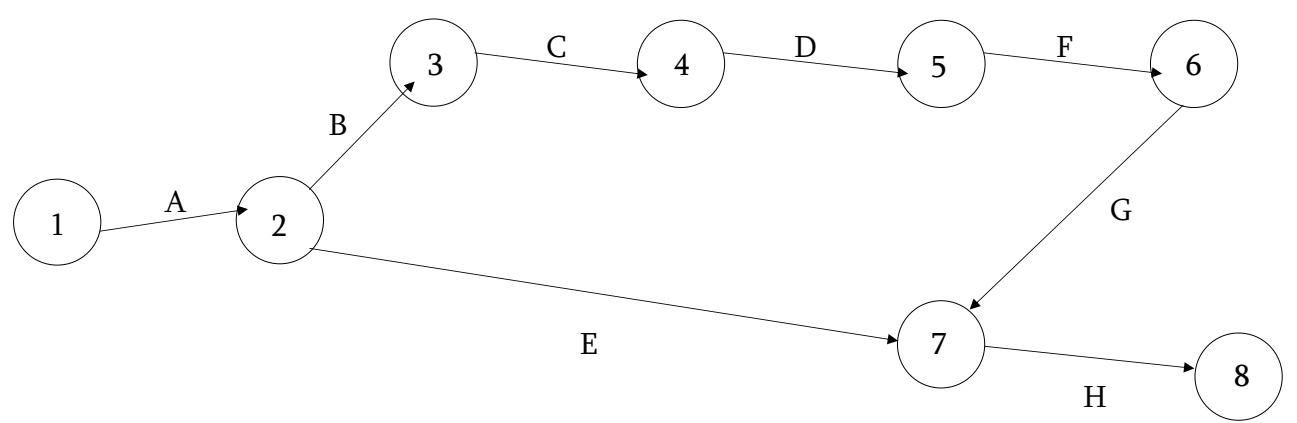

Puc. 4. Сетевой график проекта

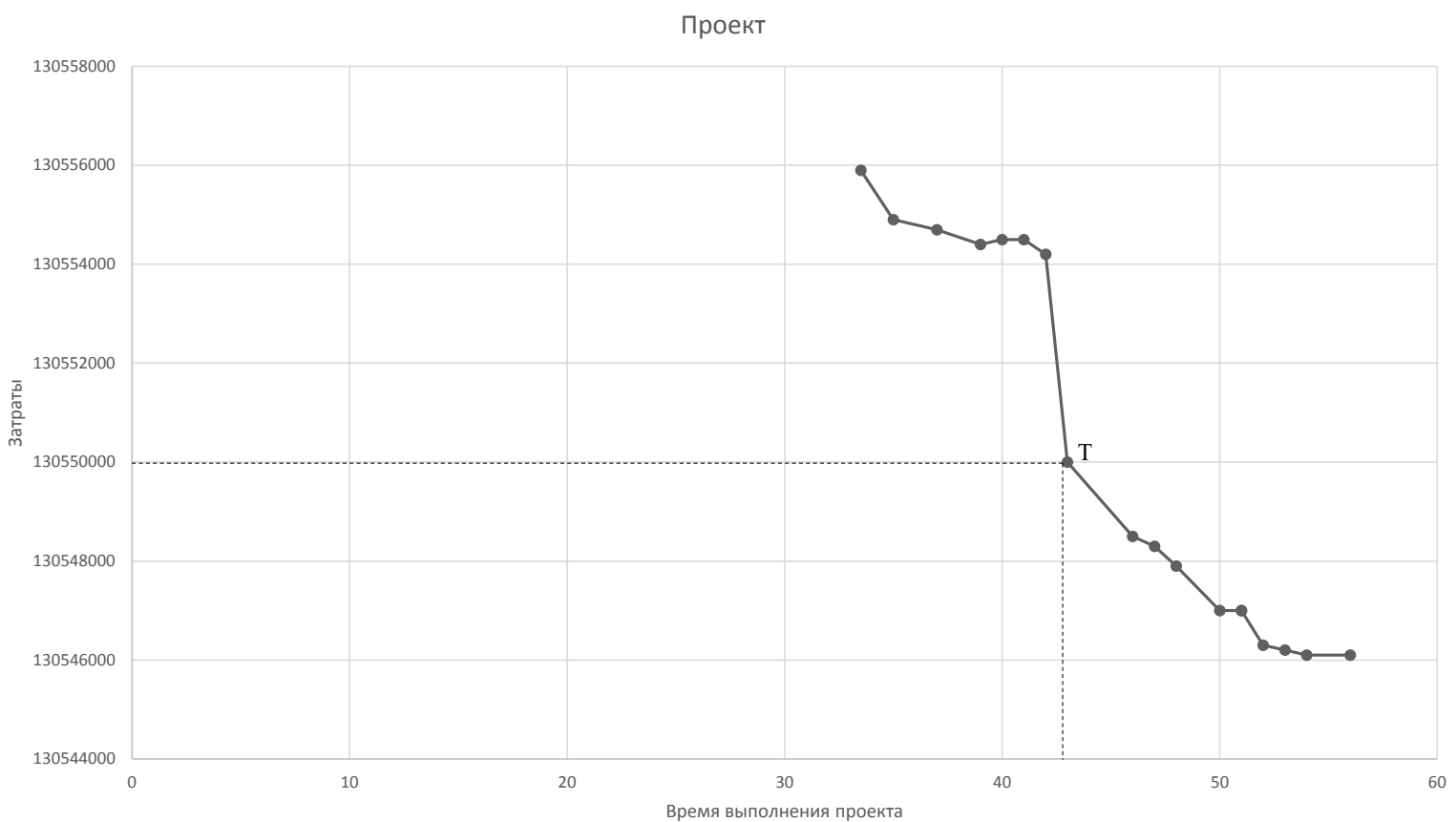

Puc. 5. Взаимосвязь затрат и продолжительности времени всего проекта

Необходимо определить крайнюю правую точку для каждой работы. Линия, которой принадлежит эта точка, является линией, используемой в процессе сокращения продолжительности работ. В начале необходимо сократить работу А до максимально возможного значения, и так далее по каждой работе.

Итоги расчётов представлены на графике взаимосвязи затрат и продолжительности времени всего проекта (рис. 5), где каждая точка соответствует совокупности исполнителей. Например, для точки Т параметры исполнения проекта - 43 месяца и 130550000 руб. При этом работу А выполняет подрядчик № 6, работу В выполняет подрядчик № 4, работу C - подрядчик № $4, \mathrm{D}-$ подрядчик № $5, \mathrm{E}-$ подрядчик № $5, \mathrm{~F}$ - подрядчик № $2, \mathrm{G}-$ подрядчик № $2, \mathrm{H}-$ подрядчик № 4.

Заказчик, определяя стоимость проекта или время, за которое он желает, чтобы проект был выполнен, может варьировать исполнителей.

Можно заметить, что при сокращении продолжительности проекта и смене подрядчиков, на графике могут появляться горизонтальные участки и даже участки убывающей функции. Подобные участки возникают из-за того, что, при переходе, подрядчик может выполнить работу, сократив и стоимость, и затраты, если это обусловлено его линией зависимости.

Поэтому на графике могут возникать точки, не оптимальные по Парето, то есть те варианты совокупностей исполнителей работ, выбор которых не является эффективным.

\section{Библиографический список}

1. Bernard W. Taylor III. Introduction to Management Science (10th Edition), Pearson, 2009, 840 pages.

2. Strader T. J., Lin F.R., Shaw, M.J. Information infrastructure for electronic virtual organization management. Decision Support Systems, 1998, № 23, p. 75-94. 